\title{
Friction Coefficient Mapping of 2D Materials via Friction-Induced Topographic Artifact in Atomic Force Microscopy
}

\author{
Thales F.D. Fernandes ${ }^{1}$ and Bernardo R. A. Neves ${ }^{1 *}$ \\ 1 Department of Physics, Universidade Federal de Minas Gerais, C.P. 702, 30123-970 Belo Horizonte, Brazil \\ Email: bernardo@fisica.ufmg.br
}

\begin{abstract}
This work explores an atomic force microscopy artifact that yields different topographic data depending on the scanning direction in contact mode. Such artifact is associated with differences in friction coefficients across the sample, which leads to up to $500 \%$ difference in topographic data of 2D materials. An analytical theory is used to explain quantitatively this artifact. Nevertheless, on the bright side, such artifact also yields a straightforward methodology, which effectively maps friction coefficients across any 2D material without the need of cantilever spring constant calibration.
\end{abstract}

Keywords: AFM; Contact-mode; friction coefficient; Euler-Bernoulli equation; 2D materials.

\section{$1 \quad$ Introduction}

Atomic Force Microscopy (AFM) is a versatile technique capable of imaging surfaces down to atomic resolution [1-3]. In contact mode (CM-AFM), it operates by employing a very sharp tip at the end of a cantilever beam and the tip-sample interaction bends the cantilever. During CM-AFM imaging, the cantilever deflection (bend angle) is maintained constant by the feedback electronics yielding a "constant normal force mode" topographic map of the sample surface [1-3]. Lateral force microscopy (LFM) is another common mode where the tip scans the sample surface perpendicularly to the cantilever axis. In such case, frictional forces twist the cantilever, making it possible to map the friction coefficients across the sample [1-3]. Frictional forces are always present in CM-AFM and, when the tip is scanned parallel to the cantilever axis, they may also induce additional cantilever deflection (bend). In such case, frictional and normal forces are mixed and the CM-AFM imaging is not a "constant normal force technique" any longer (but it still remains a constant deflection one). Such mixing of normal and frictional signals gives rise to a topographic artifact in CM-AFM images: regions with different friction coefficients will be mapped differently, even if they have the same topography. This artifact has been reported in the literature [2, 4-10], even though its analytical theory has not been presented in-depth and it has not been applied within the 2D materials framework.

Topographic artifacts may have a substantial impact on the AFM investigation of ultrathin samples, ranging from topography inversion in self-assembled monolayers [11] to more than one-hundred percent error in graphene thickness determination [12]. In this last case, Nemes-Incze et al. have reported that graphene monolayer thickness, measured using intermittent-contact mode AFM (IC-AFM), can vary more than twice its real thickness depending on the cantilever oscillation amplitude [12]. They have also performed CM-AFM measurements and have shown that a difference in thickness may occur depending on the scanning direction and that frictional forces play a non-negligible role when using CM-AFM [12]. Later, Lee et al have reported that the graphene thickness may also change depending on the sample rotation angle [13]. They have shown that the graphene thickness has a cosine dependence with the measured angle and the cantilever torsion force has a sine dependence [14]. These periodic dependences on the rotation angle were attributed to linearly aligned ripples in graphene $[5,6]$. In an earlier work, we have shown that ultrathin solid lubricants present an increase in their apparent thickness as the applied tip load is increased during CM-AFM imaging [15]. We have also shown that such negative dynamic compressibility also suffers from topographic artifacts and can be overestimated, or underestimated, depending on the scanning direction [15]. 
In view of the above paragraph, it is clear that topographic artifacts in AFM imaging are an important issue, especially with the ever-growing interest in two-dimensional (2D) materials. Therefore, in this paper, the friction-induced artifact in CM-AFM is thoroughly investigated via experiments and theory. Additionally, its application to measure friction coefficients across any 2D material sample (for example, a graphene monolayer on its supporting substrate) is also demonstrated. Finally, an analytical theory is developed to account for this artifact and its resulting friction coefficient measurement methodology.

\section{$2 \quad$ Material and Methods}

AFM experiments were carried out using a XE-70 atomic force microscope from Park Systems, Korea. All images were acquired in contact mode and in ambient conditions. Scan angles were chosen to be either parallel or perpendicular to the cantilever axis. Topographic images were processed by first order plane fits and, in all images, the substrate (silicon dioxide or gold film) was offset to zero.

CSC38 AFM probes from Mikromasch were used in all experiments (cantilever length: 345um, width: $32 \mu \mathrm{m}$, nominal thickness: $1.0 \mu \mathrm{m}$, nominal tip height: $12-18 \mu \mathrm{m}$ and nominal spring constant: $0.03 \mathrm{~N} / \mathrm{m}$ ). The real spring constant of each cantilever was calculated via the Sader method [16]. Force curves were acquired to calculate the adhesion forces, using the pull-off force, for both the substrate and the twodimensional materials investigated.

The samples consisted of mechanically exfoliated graphene or h-BN on top of a silicon wafer with a 300nm silicon dioxide layer or $\mathrm{MoS}_{2}$ on top of a gold film [9-13]. Graphene was chosen since it has a very low friction coefficient $(<0.1)[13,14]$ and is atomically thin (its measured thickness can vary from 0.35 to $1.6 \mathrm{~nm}$ depending on the tip-surface interaction and measuring technique used [12]). On the other hand, silicon dioxide has a much higher friction coefficient $(\sim 0.4-0.6)[10]$ with a gold coated silicon probe. Hexagonal boron nitride (h-BN) and molybdenum disulfide $\left(\mathrm{MoS}_{2}\right)$ samples were also investigated to show that the friction coefficient mapping methodology can be applied to any $2 \mathrm{D}$ material.

\section{$3 \quad$ Experimental Results}

Fig. 1 summarizes the results for both graphene on $\mathrm{SiO}_{2}$ (Figs. 1a - 1f) and h-BN on $\mathrm{SiO}_{2}($ Figs. $1 \mathrm{~g}-1 \mathrm{l})$. The insets at the bottom right of all images in Fig. 1 define the scanning orientation. Considering the monolayer-graphene-on- $\mathrm{SiO}_{2}$ case first (Figs. 1a - 1f), the top line of images (Figs. 1a - 1c) define the Forward scan direction and the image line below (Figs. 1d - 1f) define the Backward direction. The first two columns of images (Figs. 1a, 1b, 1d and 1e) were acquired in the parallel-to-the-cantilever-axis scanning direction, whereas the third column (Figs. 1c and 1f) was acquired in the perpendicular-to-thecantilever-axis scanning direction. The first column images (Figs. 1a and 1d) were acquired under low applied normal force, while the second and third column images were acquired under high normal force. A topographic artifact is evident in the parallel scan direction (Figs. 1a, 1b, 1d and 1e), where the apparent graphene thickness depends on both applied force and scanning direction. The graphene monolayer appears thicker in the Forward direction and thinner in the Backward one. It may even become virtually invisible (Fig. 1d) or result in topography inversion (Fig. 1e). Comparing the results of low and high normal forces, it is also evident that the artifact amplifies as the applied force increases. On the other hand, the third column of images (Figs. 1c and 1f) show that the correct graphene thickness is obtained (even at high normal forces and regardless the Forward and Backward directions) when the tip is scanned perpendicularly to the cantilever axis. In other words, the topographic artifact occurs in the parallel-to-the-cantilever-axis scanning direction only. Similar analysis can be applied to the case of a few-layer h-BN flake atop the $\mathrm{SiO}_{2}$ substrate (Figs. $1 \mathrm{~g}-1 \mathrm{l}$ ): the topography artifact occurs in the parallel scanning direction only and is force-dependent. 

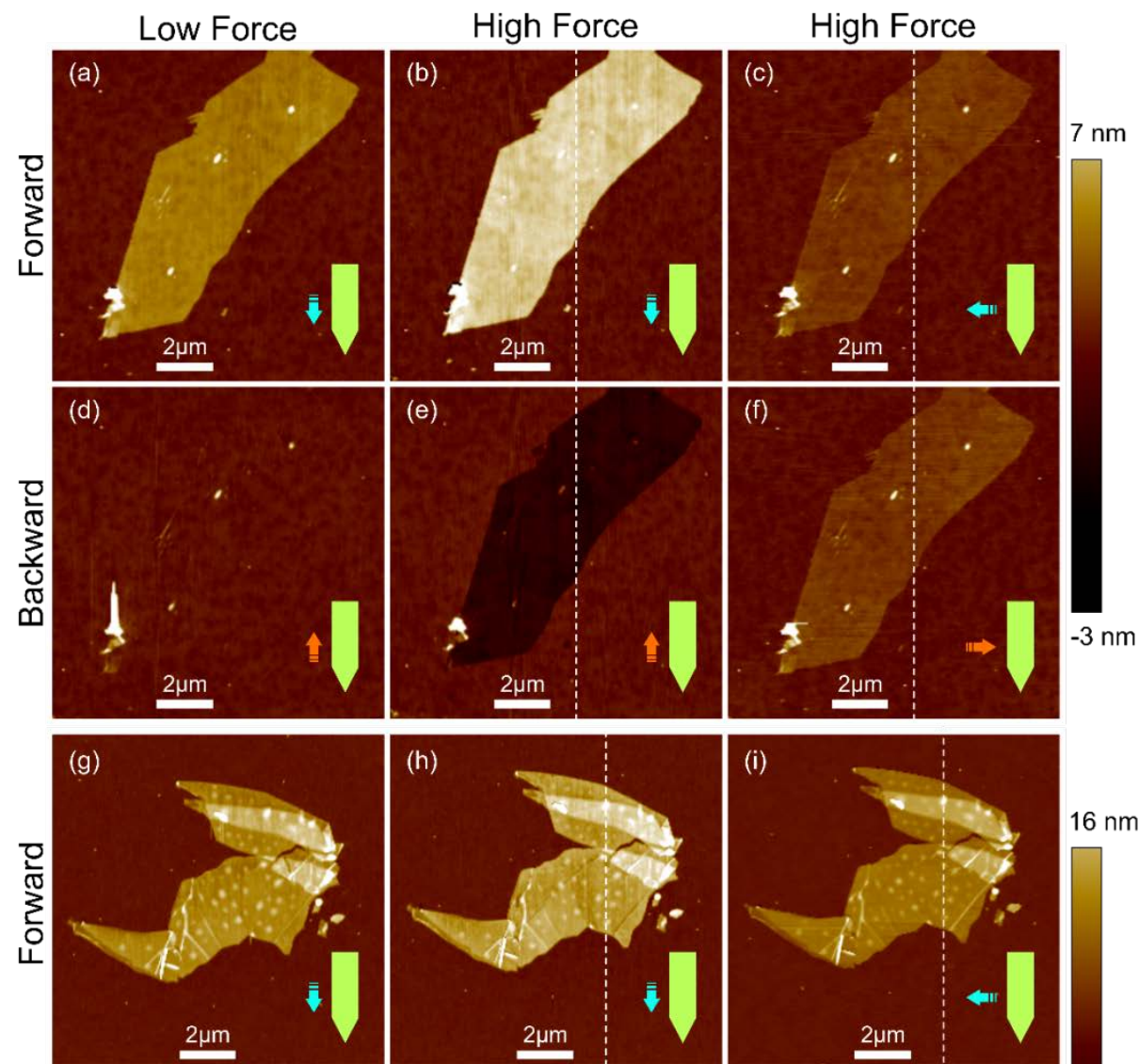

$16 \mathrm{~nm}$
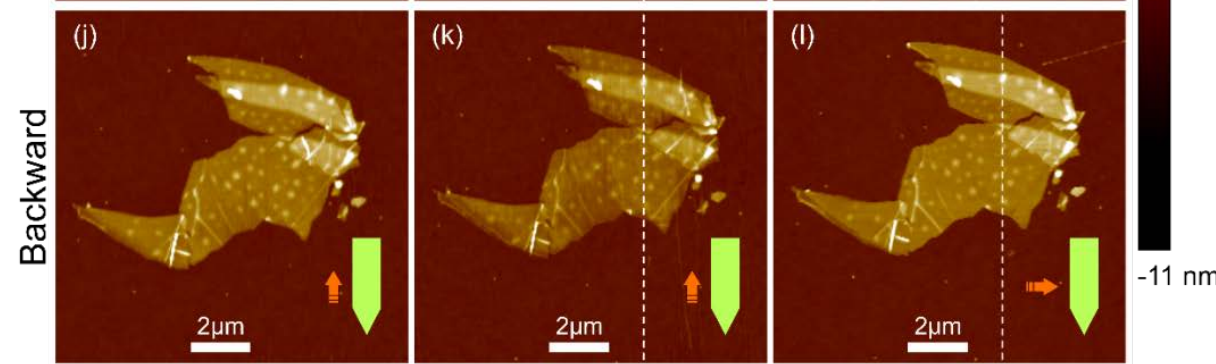

Figure 1. Topographic images of a monolayer graphene (a-f) and h-BN few-layer flake (g-l). The first column of images (Figures a, d, g and j) represents a low-force regime for the parallel geometry, the second column (Figures b, $\mathrm{e}, \mathrm{h}$ and $\mathrm{k}$ ) constitutes a high-force regime for the parallel geometry and the third column (Figures c, f, i and l) represent a high-force regime for the perpendicular geometry. The definition of Forward and Backward scanning directions is shown in the insets at the bottom right of each image, where the green symbol depicts the AFM cantilever and the red and cyan arrows indicate the scanning orientation.

Fig. 2 further illustrates this artifact by plotting line scan profiles of both graphene (Fig. 2a) and hBN (Fig. 2b) flakes in both parallel and perpendicular scanning directions (the vertical dashed lines in Figs. 1b, 1c, 1e, 1f, 1h, 1j, 1k and $1 \mathrm{l}$ indicate the location of these line profiles). For graphene (Fig. 2a), the artifact (full lines) produced more than $500 \%$ variation in the graphene monolayer thickness (achieving even negative values) when compared to the correct values obtained in the perpendicular scanning configuration (dashed lines in Fig. 2a). Similar analysis applies to the h-BN case (Fig. 2b), even though the artifact is less pronounced ( $\sim 30 \%$ variation in measured thickness) due to the larger thickness of the h-BN multilayered flake. 
(a)

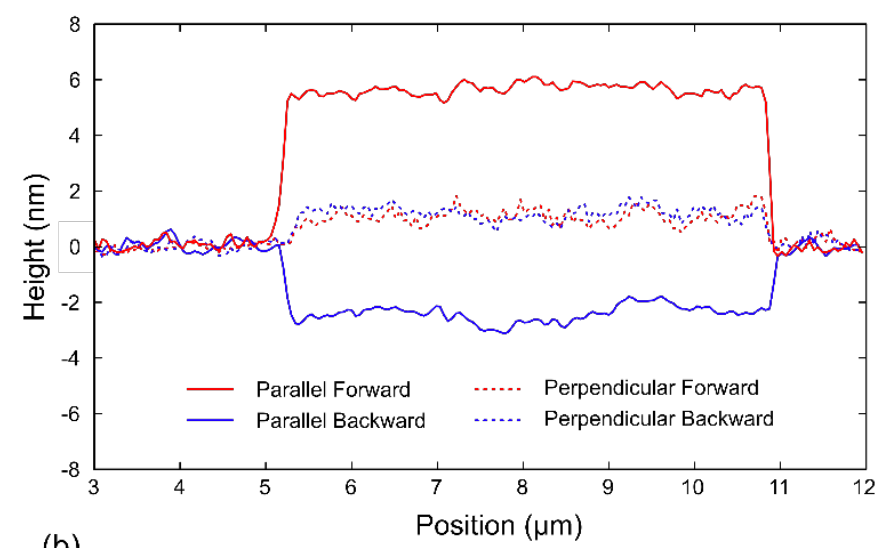

(b)

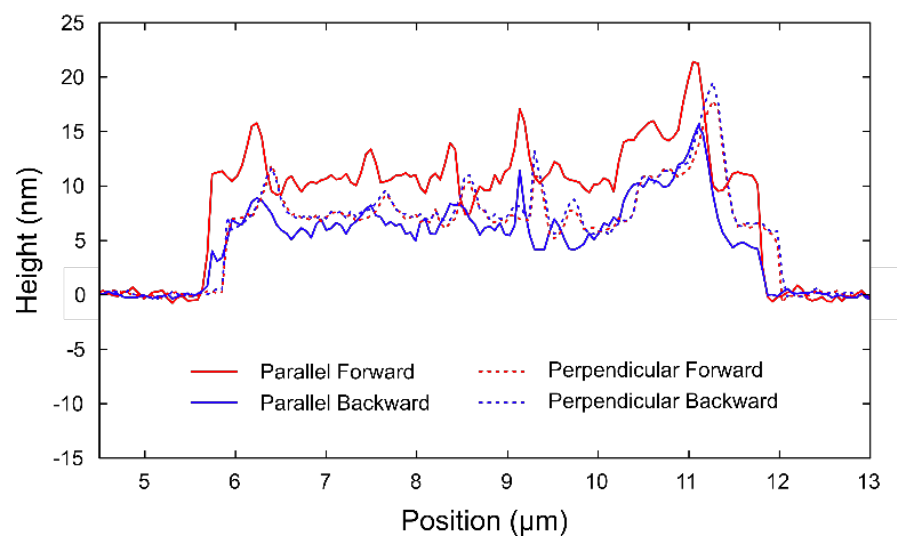

Figure 2. Line-scan profiles taken along the dotted lines in Fig. 1. Figure (a) shows the observed line profiles for graphene, while figure (b) shows line profiles for h-BN. The full lines (parallel scans) show the topographic artifact (graphene and h-BN thickness variation), whereas the dotted lines (perpendicular scans) indicate the correct topography.

The dependence of the topographic artifact with the applied normal force is illustrated in Fig. 3 for graphene monolayer. The graph plots the observed graphene thickness as a function of the applied tip load. For the perpendicular-to-cantilever-axis configuration (open triangles), the measured graphene thickness depends neither on the applied tip load nor the direction (Forward or Backward) within the force range of this experiment. However, for the parallel-to-cantilever-axis scan configuration (full triangles), the observed graphene thickness depends strongly on both applied load and scan direction (Forward or Backward). In the Forward direction, the measured graphene monolayer thickness linearly increases with the applied load (red triangles in Fig. 3), whereas the opposite, linear thickness decrease with force, occurs in the Backward direction (blue triangles in Fig. 3). In this last case, due to the small thickness of the graphene monolayer $(\sim 1 \mathrm{~nm})$, the observed thickness achieves negative values (taking the $\mathrm{SiO}_{2}$ substrate as a reference), appearing as depressions on the AFM images (see Fig. 1e). It is interesting to note in Fig. 3 that the measured thickness on the Forward and Backward directions for the parallel case does not converge to the same value at zero applied load. Linear fits of the experimental data (full lines in Fig. 3) indicate a converged thickness value of $1.5 \mathrm{~nm}$ at a force of $-13 \mathrm{nN}$. 


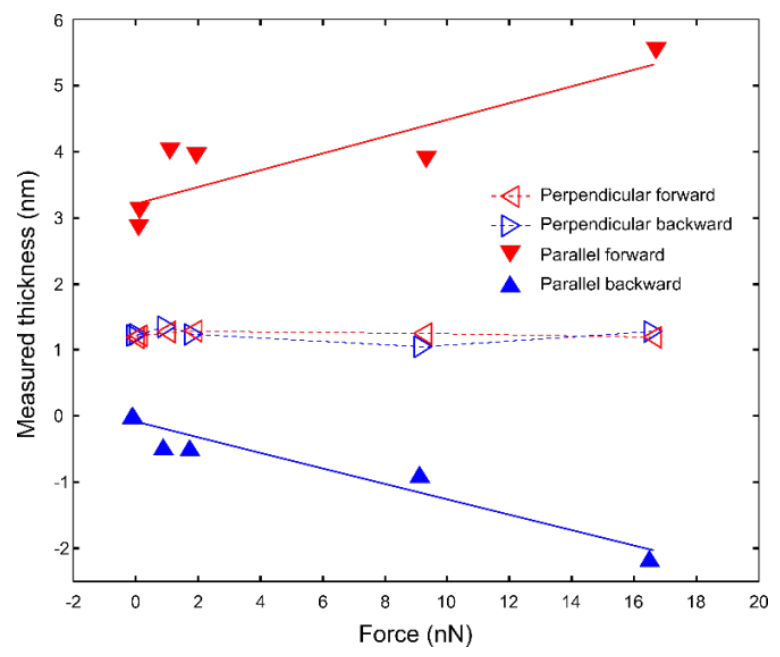

Figure 3. Experimental data of the apparent thickness of monolayer graphene as a function of the setpoint force for four different scan directions: Forward and Backward scanning directions for both perpendicular and parallel to the cantilever axis directions. The red and blue solid lines consist of a linear fit of the data, yielding $h=3.21+$ $0.13 F$ and $h=-0.088-0.12 F$, respectively, where $\mathrm{h}$ is the apparent graphene thickness and $F$ is the setpoint force.

\section{Theory: Qualitative and Quantitative}

As illustrated in Fig. 4, the experimental results of Figs. 1, 2 and 3 can be explained with a simple qualitative model of the cantilever behavior when a frictional force exists along its axis. In Fig. 4a, the cantilever is in a static situation where there are no frictional forces, an arbitrary tip load is applied and, therefore, the cantilever is bent in a concave profile. When the cantilever moves in the Backward direction, as shown in Fig. 4b, the frictional force actuates in the opposite direction. This additional force will increase the bending anticlockwise, leaving it with a more concave profile. The opposite occurs when the cantilever moves in the Forward direction as shown in Fig. 4c: the frictional force will rotate the cantilever clockwise, therefore leaving it less concave (here shown exaggeratedly as a convex deflection). Since CM-AFM is a technique of constant deflection (or constant cantilever bending), the feedback loop will raise or lower the cantilever (changing the normal force), to counteract the additional bending caused by the friction forces. As a result, in the Backward direction, the cantilever will be raised, lowering the normal force and making regions of large friction coefficient to appear thicker. In the Forward direction, the cantilever is lowered, increasing the normal force and making regions of large friction coefficient to appear thinner. Applying this rationale to the experimental results in Figs. 1, 2 and 3, graphene has a very low friction coefficient, so it will not suffer significantly from this artifact (frictional forces are small and do not change the bending significantly). However, the $\mathrm{SiO}_{2}$ substrate suffers substantially from this artifact and its measured height depends on the scanning direction (Forward or Backward). Since the graphene height (thickness) is measured relative to the $\mathrm{SiO}_{2}$ substrate, in the Backward direction, the $\mathrm{SiO}_{2}$ height increases, causing the graphene flake to appear thinner. The opposite occurs in the Forward direction: the graphene flake appears to be thicker since the $\mathrm{SiO}_{2}$ substrate is measured at a lower position. In summary, the artifact affects significantly the large friction coefficient regions. It is important to note that such effect is not an inherent property of graphene (or h$\mathrm{BN}$ ) and should appear in any sample with a difference in friction coefficients. It is more pronounced in atomically thin materials, since the artifact height, in such cases, is the same order of magnitude as the measured material. 

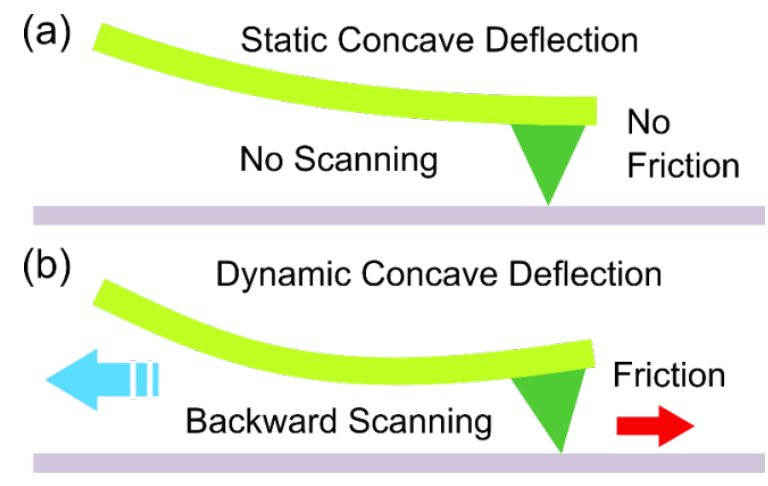

(c) Dynamic Convex Deflection

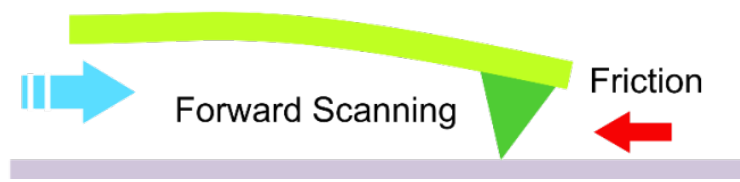

Figure 4. Qualitative picture of the topographic artifact. In (a), the cantilever is lowered to the surface while in a static situation (no scanning). Figure (b) shows a scan in the backward direction (to the left - blue arrow), where the friction force is in the opposite direction (to the right - red arrow). Figure (c) shows a scan in the forward direction (to the right) and the friction force is to the left. In figure (b), the cantilever bending is more pronounced (concave) than in (a), while in (c) it is less bent (concave) than in (a).

Besides the qualitative interpretation for the artifact presented above, a quantitative theoretical approach is also possible, which considers an analytical model of beam deflection. In such case, the beam deflection is analyzed via the Euler-Bernoulli beam equation [15], which describes it in terms of the applied load:

$$
M_{y}=-E I \frac{d^{2} w}{d x^{2}}
$$

In Eq. 1, $w$ is the beam deflection, $M$ the bending moment, $E$ the young modulus and $I$ the beam second moment of area, which can be expressed as $T^{3} W / 12$, in which $T$ is the beam thickness and $W$ its width. The bending moment $M$ depends on the beam lever arm and the applied force, and can be expressed as $\mathbf{M}=\mathbf{r} \times \mathbf{F}$. The external load $\mathbf{F}$ can be decomposed into two components: one, at the sample in-plane direction, parallel to the beam $\left(F_{x}\right)$ and another, normal to the sample $\left(F_{z}\right)$. The lever arm $\mathbf{r}$ is the distance from the point of applied load (the tip end) to the neutral axis and can be expressed as $\mathbf{r}=(L-x) \hat{\mathbf{x}}-\left(T / 2+h_{\text {tip }}\right) \hat{\mathbf{z}}$, where $L$ is the beam length and $h_{\text {tip }}$ the tip height from the apex to the base of the beam. Since the distance from the neutral axis to the tip end is needed, we define an effective tip height $h_{t i p}^{*}=T / 2+h_{t i p}$. Solving the Euler-Bernoulli equation for the case of a fixed beam $\left(w(0)=w^{\prime}(0)=0\right)$, we have:

$$
w(x)=F_{x} \frac{x^{2} h_{t i p}^{*}}{2 E I}+F_{z} \frac{(3 L-x) x^{2}}{6 E I}
$$

The second term of Eq. 2 is the basic equation used to describe the CM-AFM [23], while the additional first term describes the influence of in-plane frictional forces. Using Amonton's Law [24], the frictional forces can be expressed as $F_{x}=\mu\left(F_{z}+F_{a}\right)$, where $\mu$ is the friction coefficient and $F_{a}$ is the adhesion force [25]. For the sake of calculation simplicity, $\mu$ may assume positive or negative values: it is positive when the frictional force tends to rotate the beam anticlockwise and negative if it tends to rotate clockwise, which are the Backward and Forward directions, respectively, in accordance with Fig. 4 .

The spring constant of the cantilever $k$ is defined as the ratio of the normal force to the beam displacement when there is no friction. Hence, $k=3 E I / L^{3}$. For small deflections, the deflection angle can be expressed as $\theta(x) \approx w^{\prime}(x)$. During CM-AFM imaging, such deflection angle is set to a constant value $w^{\prime}(L)=\theta_{s}$, where $\theta_{s}$ is the angle in a static situation (no friction). From theory, it follows that 
$\theta_{s}=3 F_{s} / 2 k L$, where $F_{s}$ is the force in a static situation, also called setpoint force in CM-AFM. Therefore, the normal force $F_{z}$ can be expressed as:

$$
F_{z}=\frac{F_{s}}{1+2 \mu h_{t i p}^{*} / L}-\frac{2 \mu F_{a}}{L / h_{t i p}^{*}+2 \mu} \cong F_{s}-\left(F_{a}+F_{s}\right) \frac{2 \mu h_{t i p}^{*}}{L}+O\left(\frac{h_{t i p}^{*}}{L}\right)^{2}
$$

Where $h_{\text {tip }}^{*} / L$ is typically a small quantity $(\sim 1 / 20)$. Eq. 3 shows that normal force $F_{z}$ changes when the sign of $\mu$ changes (or the scan direction changes): $F_{z}$ is always smaller in the Backward direction (positive $\mu$ ) than in the Forward direction (negative $\mu$ ), which is in agreement with the qualitative picture of Fig. 4. Defining the artifact height $\delta h$ as the difference in deflection between the $\mathrm{SiO}_{2}$ substrate and the graphene flake for the same scanning direction (same sign of $\mu$ ) we obtain:

$$
\delta h \cong-\left(F_{s}+F_{a}\right)\left(\mu_{s}-\mu_{g}\right) \frac{h_{t i p}^{*}}{2 k L}+O\left(\frac{h_{t i p}^{*}}{L}\right)^{2}
$$

where $\mu_{s}\left(\mu_{g}\right)$ is the friction coefficient of $\mathrm{SiO}_{2}$ (graphene). Therefore, the apparent (measured) graphene height $\left(h=h_{0}+\delta h\right)$ can be expressed as:

$$
h=-F_{s}\left(\frac{h_{t i p}^{*} \Delta \mu}{2 k L}\right)+\left(h_{0}-F_{a} \frac{h_{t i p}^{*} \Delta \mu}{2 k L}\right)
$$

where $h_{0}$ is the true height (in absence of the artifact) and $\Delta \mu$ is the difference in friction coefficient.

Equation 5 describes the friction-induced artifact analytically, but it also points its useful application: the determination of friction coefficient variations $\Delta \mu$ across the sample. There is no need to know the exact value of the cantilever spring constant nor to calibrate the forces used in the AFM microscope. The latter is usually done by means of a force curve and the relationship $F_{s}=k V_{s} S$, where $S$ is the cantilever sensibility (calculated from the force curve slope) and $V_{s}$ is the voltage setpoint (measured by the photodetector). Using this relationship, Eq. 4 can be simplified to:

$$
\delta h=-\left(V_{s}+V_{a}\right) \mathrm{S} \Delta \mu \frac{h_{t i p}^{*}}{2 L}
$$

where $V_{a}$ is the adhesion force (in Volts), measured via a regular force curve. Therefore, it is possible to measure the variation in surface friction coefficients $\Delta \mu$ simply by measuring the apparent height difference $\delta h$, without any knowledge of the cantilever spring constant.

Using Eq. 6 for the same region (same frictional coefficient) but using the forward and backward scan (different signs of $\mu$ ) we have:

$$
\Delta h=-\left(V_{s}+V_{a}\right) \mathrm{S} \mu \frac{h_{t i p}^{*}}{L}
$$

where we used the fact for this case $\Delta \mu=\mu-(-\mu)=2 \mu$. Hence, it is possible to determine the frictional coefficient $\mu$ for a given material in terms of the difference in topographic images (forward/backward). Solving for the frictional coefficient in Eq. 7 we arrive:

$$
\mu=-\frac{L}{S h_{t i p}^{*}} \frac{\Delta h}{\left(V_{s}+V_{a}\right)}
$$

\section{Discussion}

The qualitative aspects of the topographic artifact were already discussed with the aid of Fig. 4. Nevertheless, a quantitative discussion is also possible using the analytical theory developed in the previous section. Using the appropriate tip parameters $\left(L, k, h_{t i p}\right)$ into Eq. (5) and equaling it to the fits of experimental data in Fig. 3, we can obtain the friction coefficient variation $\Delta \mu$ and adhesion force $F_{a}$, which yields $\Delta \mu=0.31, F_{a}=16 n N$ in the Forward scan and $\Delta \mu=0.29, F_{a}=11 n N$ in the Backward scan. Such friction coefficient variation $\Delta \mu \sim 0.3$ between the $\mathrm{SiO}_{2}$ substrate and graphene is in good agreement with the data in the literature [20,21], which corroborates our model. The average adhesion force $F_{a}$ measured from both Forward and Backward directions is $F_{a}=13.5 n N$, which is in excellent agreement with the experimental value for the measured adhesion force $F_{a}=13.3 n N$. Returning to the 
last paragraph of section 3 - Experimental Results - it was shown that the linear fits in Fig. 3 for both Forward and Backward directions would converge to the correct graphene thickness at a negative force $F=-13 n N$. Such negative force is, by definition, the adhesion force $F_{a}$, which was measured $\left(F_{a}=13.3 n N\right)$ and independently calculated from Eq. $5\left(F_{a}=13.5 n N\right)$. The good agreement between all these values gives further support to the present model of the topographic artifact.

The experimental results in Fig. 1, 2 and 3 also show that this artifact does not occur in the perpendicular-to-the-cantilever-axis scanning direction. The reason is that a torsional force does not change the cantilever bending, as verified by finite-element analysis of cantilever twist (data not shown). Therefore, there is no further deflection caused by friction forces in the perpendicular configuration and LF-AFM remains free of this kind of artifact. Additionally, using the experimental data in Figs. 1b and 1e (as an example) together with Eq. 6 , the friction coefficient variation $\Delta \mu$ can be directly estimated from the graphene height variation, yielding $\Delta \mu=0.31$, which again is in good agreement with the literature and illustrates an interesting feature of this topographic artifact.

Table 1. Frictional coefficient of $2 \mathrm{D}$ materials and frictional coefficient variation from the substrate.

\begin{tabular}{lcc}
\hline \multicolumn{1}{c}{ Material } & $\begin{array}{c}\text { Frictional } \\
\text { coefficient }(\mu)\end{array}$ & $\begin{array}{c}\text { Frictional coefficient } \\
\text { variation }(\Delta \mu)\end{array}$ \\
\hline Graphene (on top $\left.\mathrm{SiO}_{2}\right)$ & 0.053 & 0.31 \\
$\mathrm{~h}-\mathrm{BN}$ (on top $\left.\mathrm{SiO}_{2}\right)$ & 0.22 & 0.15 \\
$\mathrm{MoS}_{2}$ (on top $\mathrm{Au}$ ) & 0.071 & 0.22 \\
$-\mathrm{SiO}_{2}$ (from graphene) & 0.34 & - \\
$\mathrm{SiO}_{2}$ (from h-BN) & 0.32 & - \\
$\mathrm{Au}$ & 0.28 & - \\
\hline
\end{tabular}

Finally, the most useful application of this artifact is described by Eq. 8: it is possible to calculate the absolute frictional coefficient $\mu$ (with the AFM tip material) of each individual sample material (2D flake and its supporting substrate) simply by measuring its apparent height variation on forward and backward scans. In order to illustrate its applicability to other substrates and 2D materials, a third sample type $\left(\mathrm{MoS}_{2}\right.$ on $\left.\mathrm{Au}\right)$ was prepared and measured. Therefore, Table 1 summarizes this methodology showing the absolute frictional coefficient $\mu$ for all 2D materials and substrates investigated. The frictional coefficient variation $\Delta \mu$ between a $2 \mathrm{D}$ flake and its supporting substrate, as calculated from Eq. 6, is also shown in Table 1. As an example, the graphene frictional coefficient is $\mu$ $=0.053$, hence the variation calculated above is mainly due to the $\operatorname{SiO}_{2}(\mu=0.34)$. Both methods derived from Eqs. 6 and 8 (variation of frictional coefficient and absolute frictional coefficient, respectively) agree well with each other, corroborating their strength.

\section{Conclusions}

In this work, a topography artifact that occurs in CM-AFM when the tip is scanned parallel to the cantilever axis is analyzed. Although such artifact may be initially regarded as a deleterious feature, due to the mismatch between Forward and Backward topography channels, it is shown that it can be used to measure the friction coefficients and adhesion forces of a sample. This simple method of friction constant determination does not require spring constant values (either normal or torsional) of the cantilever. The need of such values is a major drawback of LF-AFM, which requires the measurement of both normal and torsional spring constants. Although there are several methods for spring constant calibration [1-3, 16], they are precise, typically, within $10 \%$ and error propagation can result in even larger errors in quantitative friction values [1-3]. Additionally, calibration of the photodetector lateral signal is also required for quantitative LF-AFM, which demands special reference samples and a tedious procedure [2]. Therefore, the simplicity of the present method actually adds robustness to the task of friction coefficient determination.

The results also show that such artifact does not occur when the tip scans the sample perpendicularly to the cantilever axis since torsional forces do not produce additional bending of the cantilever. Therefore, such scanning orientation should be adopted when the correct sample height (thickness) is to 
be measured in CM-AFM. Finally, the observed artifact is not an intrinsic property of the investigated 2D materials but should occur in any surface with different friction coefficients. In other words, the friction-induced artifact is a general property of any system with friction variations across its surface and its quantification should enable the widespread application of the friction coefficient methodology explored in this work.

Acknowledgments. This work was supported by CNPq, Fapemig and INCT Nanocarbono, Brazil.

\section{References}

1. V. L. Mironov, "The Fundamentals of Scanning Probe Microscopy," 2004.

2. B. Bhushan, "Nanotribology and Nanomechanics: An Introduction," Springer, 2005.

3. P. Eaton and P. West, "Atomic Force Microscopy," Oxford University Press, 2010.

4. B. R. A. Neves, D. N. Leonard, M. E. Salmon, P. E. Russell, and E. B. Troughton, "Observation of topography inversion in atomic force microscopy of self-assembled monolayers," Nanotechnology, vol. 10, no. 4, pp. 399404,1999.

5. P. Nemes-Incze, Z. Osváth, K. Kamarás, and L. P. Biró, "Anomalies in thickness measurements of graphene and few layer graphite crystals by tapping mode atomic force microscopy," Carbon, vol. 46, no. 11, pp. 435-1442, 2008.

6. D. H. Lee, M. J. Lee, Y. S. Kim, J. S. Choi, and B. H. Park, "Sample rotation angle dependence of graphene thickness measured using atomic force microscope," Carbon, vol. 81, pp. 210-215, 2015.

7. J. S. Choi et al., "Facile characterization of ripple domains on exfoliated graphene," Review of Scientific Instruments, vol. 83, no. 7, pp. 73905, 2012.

8. A. P. M. Barboza et al., "Dynamic Negative Compressibility of Few-Layer Graphene, h-BN, and MoS2," Nano Letters, vol. 12, no. 5, pp. 2313-2317, 2012.

9. J. E. Sader, J. W. M. Chon, and P. Mulvaney, "Calibration of rectangular atomic force microscope cantilevers," Review of Scientific Instruments, vol. 70, no. 10, pp. 3967, 1999.

10.K. S. Novoselov, "Electric Field Effect in Atomically Thin Carbon Films," Science, vol. 306, no. 5696, pp. 666669, 2004.

11.N. Alem, R. Erni, C. Kisielowski, M. D. Rossell, W. Gannett, and A. Zettl, "Atomically thin hexagonal boron nitride probed by ultrahigh-resolution transmission electron microscopy," Physical Review B, vol. 0, no. 15, pp. $155425,2009$.

12.L. Song et al., "Large Scale Growth and Characterization of Atomic Hexagonal Boron Nitride Layers," Nano Letters, vol. 10, no. 8, pp. 3209-3215, 2010.

13.D. Berman, A. Erdemir, and A. V. Sumant, "Graphene: a new emerging lubricant," Materials Today, vol. 17, no. 1, pp. 31-42, 2014.

14.Y. J. Shin et al., "Frictional characteristics of exfoliated and epitaxial graphene," Carbon, vol. 49, no. 12, pp. 4070-4073, 2011.

15.J. M. Gere and S. P. Timoshenko, "Mechanics of Materials, 2nd ed.," Wadsworth Publishing Co Inc, 1984.

16.D. Sarid, "Scanning Force Microscopy: With Applications to Electric, Magnetic, and Atomic Forces," New York Oxford, 1991.

17.Y. Mo, K. T. Turner, and I. Szlufarska, "Friction laws at the nanoscale," Nature, vol. 457, no. 7233, pp. 11161119, 2009 .

18.D. L. Sedin and K. L. Rowlen, "Adhesion Forces Measured by Atomic Force Microscopy in Humid Air," Analytical Chemistry, vol. 72, no. 10, pp. 2183-2189, 2000. 